\title{
Analysis of Surgery Types, Length of Stay, and the Needs of Post-Elective Surgery ICU Before and During the COVID-19 Pandemic
}

\author{
Juanita Deifa Pricillia Tetengean ${ }^{1 *}$, Bambang Pujo Semedi ${ }^{1}$, Edward Kusuma ${ }^{1}$ \\ 1 Department of Anesthesiology and Intensive Care, Faculty of Medicine Airlangga University, \\ Dr Soetomo General Hospital, Surabaya, Indonesia \\ *Corresponding Author: Juanita Deifa Pricillia Tetengean \\ E-mail: juanitatetengean79@gmail.com
}

Abstract

Background: The COVID-19 pandemic led to a reduction in elective surgeries by assessing the urgency of surgeries and surgical rooms being converted into additional ICUs. This study aimed to analyze the selection of surgery types, length of stay, and the needs of post-elective surgery ICU before and during the COVID-19 pandemic at Dr. Soetomo General Hospital Surabaya.

Methods: The study was an observational analytic study, with a retrospective cohort design. Subjects were adult patients who require elective surgeries at Dr. Soetomo General Hospital in March 2019 - February 2021. Data on the type of surgery, length of stay, and needs of ICU after elective surgery were obtained from medical records. Statistical analysis was done using comparative tests according to the type of research data.

Results: A total of 8971 patients were included in the study. There was significant differences in the distribution of the types of surgeries $(p=<0.001)$ and ICU needs $(p=0.017)$ before and during the pandemic. The total surgeries were reduced by $41.4 \%$. The needs for ICU increased from $1 \%$ before the pandemic to $1.6 \%$ during the pandemic. There was no significant difference in the distribution of length of stay before and during the pandemic $(\mathrm{p}=0.978)$.

Conclusion: There were differences in the number of types of operations and the needs of post-elective surgery ICU before and during the COVID-19 pandemic. But there was no difference in the length of stay of patients after elective surgery before and during the COVID-19 pandemic.

\section{Introduction}

The COVID-19 pandemic has brought out a crisis that has not existed since the post-war era, namely an exponential increase in the number of patients requiring sub-intensive or intensive care in almost all health centers around the world [1]. The pandemic has also caused various policy changes, such as changes of the operating schedule due to the hospital being overwhelmed, the operating room being transformed to an 
additional ICU, and non-urgent surgical procedures being cancelled. Medical and paramedical staff were relocated to provide support for patients with COVID-19 infection [2].

Planning a surgery now has to take into account the more limited access to operating rooms (many have been converted into ICUs) and that the risk of spreading the virus persists even in the operating theatres, leading to various policy changes including reducing the number of elective surgeries by assessing the urgency of surgeries [3]. According to available data, SARS-CoV-2 is thought to be spread mainly through respiratory droplets and close contact. However, because this mode of transmission cannot explain all cases, there is still the possibility that airborne transmission can occur [4].

The COVID-19 pandemic has caused many patient visits to be postponed, non-emergency surgery delays, hospitalization time affected by additional examinations and bed occupancy rates, and increased costs for performing procedures caused by additional examinations. However, data regarding the impact of the COVID19 pandemic in Indonesia on the issues mentioned are still very limited, although such data is needed to determine a better strategy during this pandemic [5]. Therefore, this study was made to determine the differences in the type of surgery, length of stay, and post-operative ICU requirements before and during the COVID-19 pandemic at Dr. Soetomo General Hospital Surabaya, so that later when faced with a pandemic, the hospital already has a strategy and data on how many percent of elective surgeries must be reduced and what percentage of the ICU needs are needed.

\section{Methods}

The study was an observational analytic study, with a retrospective cohort design. Sampling technique used was the total sampling technique. Inclusion criteria were adult patients, over the age or equal to 17 years of age who require elective surgery at Dr Soetomo General Hospital in March 2019 - February 2021. The study was conducted in March 2019 to February 2021 (Pre-pandemic era: March 2019 to February 2020, pandemic era: March 2020 to February 2021). Data on the type of surgery, length of stay, and needs of ICU after elective surgery were obtained from medical records documents. Statistical analysis was done using comparative tests according to the type of research data. The level of significance used was $5 \%, p$ value $<0.05$ was considered significant.

\section{Results}

\subsection{Characteristics of Research Sample}

A total of 8971 patients met the inclusion and exclusion criteria which were divided into two groups, namely patients who underwent surgery before and during the COVID-19 pandemic. The general characteristics of the study sample were assessed by age as shown in table 1 .

In this study, there was a significant decrease in the number of patients during the pandemic $(p=0.011)$. There was no change in the proportion before and during the pandemic for both men and women. Furthermore, statistical analysis with Mann-Whitney test was carried out to examine the age variable in the group before and during the pandemic, it can be concluded that there were differences in the age distribution before and during the pandemic with $\mathrm{p}$ value of 0.006 . There was a significant decrease in the number of all age groups during the pandemic. In addition, it was found that the age distribution tends to be smaller during the pandemic than before the pandemic by looking at the average age value. 
Table 1. Characteristics of Research Sample

\begin{tabular}{lcc}
\hline Variable & \multicolumn{2}{c}{ Patients (n: 8971) } \\
\cline { 2 - 3 } Gender $-\mathbf{N}(\%)$ & Before pandemic & During pandemic \\
Male & $3795(59.8 \%)$ & $1497(56.9 \%)$ \\
Female & $2547(40.2 \%)$ & $1132(43.1 \%)$ \\
\hline Age (year) & $46.63 \pm 15.900$ & $45.65 \pm 15.514$ \\
$\mathbf{< 1 8}$ & 87 & 35 \\
$\mathbf{1 8}-\mathbf{3 0}$ & 1177 & 483 \\
$\mathbf{3 1 - 4 0}$ & 977 & 493 \\
$\mathbf{4 1 - 5 0}$ & 1323 & 542 \\
$\mathbf{5 1 - 6 0}$ & 1451 & 588 \\
$\mathbf{7 0}$ & 1327 & 488 \\
\hline
\end{tabular}

3.2. Data of Surgery Types Before and During COVID-19 Pandemic

The types of elective surgeries in this study were categorized based on the data listed in the medical record. Types of surgeries as shown in table 2 .

Table 2. Elective Surgery Types

\begin{tabular}{lccc}
\hline \multirow{2}{*}{ Surgery types } & \multicolumn{2}{c}{ Patients (n: 8971) } & $\begin{array}{c}\text { Percentage of } \\
\text { Decrease }(\%)\end{array}$ \\
\cline { 2 - 4 } Neurosurgery & Before Pandemic & During Pandemic & $54.1 \%$ \\
\hline Urology & 289 & 86 & $47.4 \%$ \\
\hline Dental \& Oral Surgery & 734 & 262 & $46.1 \%$ \\
\hline Ophtalmology & 141 & 52 & $45.9 \%$ \\
\hline Obstetry \& Gynecology & 707 & 262 & $43.0 \%$ \\
\hline Plastic Surgery & 793 & 316 & $42.7 \%$ \\
\hline Head \& Neck Surgery & 259 & 104 & $39.9 \%$ \\
\hline General Surgery & 556 & 239 & $39.8 \%$ \\
\hline Orthopaedic \& Traumatology & 1792 & 772 & $37.0 \%$ \\
\hline Thorax \& Cardiovascular & 959 & 441 & $8.2 \%$ \\
\hline Total & 112 & 95 & $41.4 \%$ \\
\hline
\end{tabular}

The types of surgeries were categorized into 10 groups. The more common types of surgeries performed were General Surgery (2564 procedures), Orthopedic \& Traumatology Surgery (1400) and Obstetrics \& Gynecology (1109). Then statistical analysis was carried out with the Chi-square test and obtained $\mathrm{p}$ value of $<0.001$ which indicated a significant difference to the distribution of the types of surgeries data before and during the pandemic.

The largest reductions in the number of surgeries were neurosurgery, urology, and dental-oral surgery $(54.1 \%, 47.4 \%$, and $46.1 \%$, respectively). The least reduction in the number of surgeries was thoraxcardiovascular surgery, which was $8.2 \%$. Generally, the reduction in the total number of surgeries during the pandemic was $41.4 \%$. 


\subsection{Length of Stay Before and During COVID-19 Pandemic}

Data on length of stay before and during the pandemic are shown in table 3.

Table 3. Length of Stay

\begin{tabular}{lccc}
\hline Variable & \multicolumn{2}{c}{ Patients (n: 8971) } & p \\
\cline { 2 - 3 } & Before Pandemic & During Pandemic & \\
\hline LOS & $9.41 \pm 6.407$ & $9.30 \pm 5.705$ & 0.978 \\
\hline
\end{tabular}

The result of the normality test for length of treatment showed that the data was not normally distributed ( $p=0.000$ ), so that statistical test was carried out using Mann-Whitney test, the $p$ value was 0.978. It was concluded that there was no significant difference in the distribution of LOS before and during pandemic.

\subsection{Non COVID-19 ICU Requirements Post-Elective Surgery Before and During COVID-19 Pandemic}

After the surgeries were carried out, further observations were made on the patients' clinical condition whether they require a non COVID-19 ICU or not. The non COVID-19 ICU needs that were observed were based on the data from the medical records, as listed in table 4.

Table 4. ICU Requirements Post-Elective Surgery

\begin{tabular}{|c|c|c|c|}
\hline \multirow{2}{*}{ Variable } & \multicolumn{2}{|c|}{ Patients (n: 8971) } & \multirow[b]{2}{*}{$\mathbf{p}$} \\
\hline & Before Pandemic & During Pandemic & \\
\hline \multicolumn{4}{|c|}{ ICU Requirements } \\
\hline Yes & $62(1.0 \%)$ & $42(1.6 \%)$ & 0.017 \\
\hline No & $6280(99.0 \%)$ & $2587(98.4 \%)$ & \\
\hline
\end{tabular}

The data for non COVID-19 ICU needs then statistically analyzed with the Chi-square test, and the results obtained was $\mathrm{p}$ value of 0.017 . It could be concluded that there was a significant difference in the distribution of non COVID-19 ICU needs before and during the COVID-19 pandemic. In the pre-pandemic period, the needs for ICU was $1.0 \%$ and increased during the pandemic to $1.6 \%$, which means there was a $0.6 \%$ increase in the needs for ICU.

\section{Discussion}

\subsection{Characteristics of Research Subjects}

In this study, all patients who underwent elective surgery before and during the pandemic were included in the study sample. The period before the pandemic started from March 2019 to February 2020, while the period during the pandemic was from March 2020 to February 2021. A total of 8971 patients met the inclusion and exclusion criteria, of which 6342 patients were in the pre-pandemic period and 2629 patients were during the pandemic.

In the pre-pandemic group, the median age of the patients was $46.63 \pm 15.900$, while the median age of the patients during the pandemic was $45.65 \pm 15.514$. This is similar to other studies, which were conducted on elective surgery patients in the surgical oncology division before and during the early phase of the COVID-19 pandemic at M Djamil Hospital Padang where the mean age of patients before the pandemic was 48.72 years 
and the initial phase of the pandemic was 49.53 years [6]. The results in this study were different from the results of other studies, where in previous study an analysis of the clinical characteristics of patients undergoing elective surgery in the early period of the pandemic, the results obtained that the average age of patients was 55 years (IQR = 43-63) [7]. In this study, based on statistical analysis, p value of 0.006 indicated a difference in the age distribution of patients before and during the pandemic.

\subsection{Types of Surgery Before and During COVID-19 Pandemic at Dr Soetomo General Hospital Surabaya}

The types of surgeries observed in this study were all elective surgeries. In a study that analyzed surgical cases at 170 hospitals in the United States during the COVID-19 pandemic, it was found that the number of cases decreased from 86,004 at the beginning of the pandemic to 15,699 cases. If calculated as a percentage, then the decrease was $81 \%$ [8]. Another study also noted a rapid decline in elective procedures at the start of the pandemic [9].

The elective surgery schedule is affected by the COVID-19 pandemic. The postponement of elective surgery during a pandemic is one of the fundamental steps to maintain patient safety and limit the spread of the virus. This measure increases human resources and tools to treat COVID-19 patients, increasing bed capacity in wards and intensive care units (ICUs). In addition, avoiding unnecessary patient traffic in hospitals and reducing the risk of cross-infection between elective patients, hospital visitors and COVID-19 patients, as well as preventing the spread of infection from the hospital to the community [10].

Delaying surgeries can affect the mental health of the patients. Previous study was conducted in the United States on 47 patients who had delayed cardiovascular surgeries between March 13 and June 1, 2020 due to the COVID-19 pandemic. Patients were interviewed by the research team to explore the patients' emotions and perspectives regarding the postponement of their surgeries. From the results of the interview, it was found that patients felt that the opportunity to return to a "normal" life was lost and failed to improve the expected quality of life. In the study, physical suffering accompanied by emotional disturbances resulted in a decrease in the general condition of the patient. In some patients with poor coping mechanisms, this can trigger the idea of ending life/suicide [11].

In delaying elective surgery, it is necessary to carry out a risk and benefit assessment. Surgical strategies during a pandemic need to continually adapt to evolving situations. In particular, surgical planning must take into account local resource constraints as a way of understanding the potential impact of each decision. Dr Soetomo General Hospital Surabaya took a policy step by limiting the number of operations during the pandemic. Letter number 605/71/301.2.8/IBP/V/2020 regarding the Efficiency of the Operating Room was issued by the Head of the Central Surgical Installation of Dr. Soetomo General Hospital. The letter stated that there was a $71 \%$ reduction in elective surgery activities due to the COVID-19 pandemic and the mobilization of nurses to concentrate on COVID-19 patient care. Operating room efficiency was carried out by closing 8 out of 22 operating rooms at Dr. Soetomo General Hospital. A total of 14 operating rooms operated for all surgical departments in Dr. Soetomo General Hospital. Each surgical department gets a portion of 1 operating room.

The data on the highest number of elective surgeries before the pandemic was general surgery with 1792 cases, and when the COVID-19 pandemic hit the most elective surgeries were still from the same division with 772 cases. In contrast to the study by Prasad et al, the most common type of selective surgery performed was ophthalmological surgery (1537.8 cases/week) in the pre-pandemic period, while during the pandemic musculoskeletal surgery (891.3 cases/week) [8]. When viewed from the percentage reduction in the number of operations, operations in the field of neurosurgery have the highest percentage, while thoracic-cardiovascular surgery has the lowest percentage reduction. 
In this study, neurosurgery was the type of surgery that experienced the greatest decrease in numbers during the pandemic. Neurosurgery is one of the operations that requires a lot of hospital resources (surgery lasts a long time and requires intensive care after surgery). Meanwhile, thoracic-cardiovascular surgery experienced the least decrease. This is because the type of patient's disease in the thoracic-cardiovascular field has an urgent nature/needs immediate surgery. In a study conducted in Brussels, Belgium, the greatest reduction in elective surgery was found in general surgery types. In this study, digestive and vascular surgery were included in the general surgery category. Meanwhile, the one that experienced the least decrease was plastic surgery [12].

\subsection{Length of Stay Before and During COVID-19 Pandemic at Dr Soetomo General Hospital Surabaya}

The mean length of stay for patients in this study before the pandemic was 9.41 days, while during the pandemic it was 9.30 days. After statistical analysis, the $p$ value was 0.978 , so it was concluded that there was no significant difference in the distribution of length of stay before and during the pandemic.

\subsection{ICU Requirements Post Elective Surgery Before and During COVID-19 Pandemic at Dr Soetomo General Hospital Surabaya}

There was a significant difference in the distribution of non COVID-19 ICU needs after elective surgery between before and during the pandemic, with $\mathrm{p}$ value of 0.017 , where before the pandemic $1 \%$ (62 patients) required treatment in the ICU, and 1.6\% (42 patients) during the pandemic. In other study, it was stated that out of a total of 77,640 cases of elective procedures, 2865 (4\%) cases required ICU treatment, while during the pandemic 7,007 elective procedures were carried out, of which 534 (8\%) required admission to the ICU [8].

In this study, the number of ICU needs increased slightly $(0.6 \%)$ as seen from the percentage of the total number of surgical cases. The increase in the percentage of ICU needs during the pandemic can be related to policies in choosing the type of elective surgery. The type of surgery chosen is urgent/immediate, where the action is usually high risk with the patient's condition being less than optimal. However, the proportion of ICU needs for these cases remained between before and during the pandemic.

\section{Conclusion}

There were differences in the number of types of operations and the needs of post elective surgery ICU before and during the COVID-19 pandemic. But there was no difference in the length of stay of patients after elective surgery before and during the COVID-19 pandemic.

\section{Conflict of Interests}

None.

\section{Acknowledgements}

None. 


\section{References}

1. de Simone, B., Chouillard, E., di Saverio, S., Pagani, L., Sartelli., M., Biffl, W. L., et al. Emergency surgery during the COVID-19 pandemic: What you need to know for practice. Annals of the Royal College of Surgeons of England, 2020;102(5), pp. 323-332.

2. Stahel, P. F. How to Risk-Stratify Elective Surgery during the COVID-19 Pandemic?. Patient Safety in Surgery, 2020;14(8), pp. 1-4.

3. Burhan, E., Susanto, A. D., Nasution, S. A., Ginanjar, E., Pitoyo, C. W., Susilo, A., et al. Pedoman Tatalaksana COVID-19, 3 ed. 2020.

4. Dick, L., Green, J., Brown, J., Kennedy, E., Cassidy, R., Othman, S., et al. Changes in Emergency General Surgery During Covid-19 in Scotland: A Prospective Cohort Study. World Journal of Surgery, 2020;44(11), pp. 3590-3594.

5. Ljungqvist, O., Nelson, G. and Demartines, N. The Post COVID-19 Surgical Backlog: Now is the Time to Implement Enhanced Recovery After Surgery (ERAS). World Journal of Surgery, 2020;44(10), pp. 31973198.

6. Fitra, M., Harahap, W. A., Zulfiqar, Y. Elective surgery service on oncology surgery division before and during early pandemic era of Corona virus disease 19 (COVID-19) in Dr M Djamil Hospital Padang. Biomedical Journal of Indonesia, 2021;7(1), pp. 181-192.

7. Lai, C. C., Shih, T. P., Ko, W. C., Tang, H. J., Hsueh, P. Severe acute respiratory syndrome coronavirus 2 (SARS-CoV-2) and coronavirus disease-2019 (COVID-19): The epidemic and the challenges. International Journal of Antimicrobial Agents, 2020;55(3), p. 105924.

8. Prasad, N. K., Englum, B. R., Turner, D. G., Lake, R., Siddiqui, T., Mayorga-Carlin, M. A nation-wide review of elective surgery and COVID-Surge capacity, Journal of Surgical Research, 2020;267, pp. 211216.

9. Uimonen, M., Kultunen, I., Paloneva, J., Launonen, A. P., Ponkilainen, V., Matilla, V.M. The impact of the COVID-19 pandemic on waiting times for elective suregery patients : A multicenter study. Plos One, 2021;16(7), p. e0253875.

10. Moletta, L., Pierobon, E. S., Capovilla, G., Costantini, M., Salvador, R., Merigliano, S., et al. International guidelines and recommendations for surgery during Covid-19 pandemic: A Systematic Review. International Journal of Surgery, 2020;79(May), pp. 180-188.

11. Byrnes, M. E., Brown, C. S., de Roo, A. C., Corriere, M. A., Romano, M. A., Fukuhara, S., et al. Elective Surgical Delays Due to COVID-19: The Patient Lived Experience. Medical Care, 2021;59(4), 288-294.

12. Firmansyah, M. I., Rahmanto, F. and Setiawan, D. The Preparedness for the Covid-19 Pandemic Management in Indonesia. Jurnal Administrasi Kesehatan Indonesia, 2020;8(2), p. 188. 